pendant 30 minutes serait suffisante pour rendretous ces organismes non viables.

Plusieurs expérimentateurs ont étudié les pasteurisateurs industriels. Sмптн (1932) ne put obtenir Brucella abortus sur 187 échantillons traités par pasteurisation lente de 30 minutes à $145^{\circ} \mathrm{F}$. (62077 C.). Murray, MoNutw et Purwin (1932) ont trouvé que les pasteurisateurs à cuve ne sont pas parfaits, à moins qu'ils ne fonetionnent bien. Des espèces bovine et porcine survivaient à des pasteurisations de 30 minutes à $140^{\circ} \mathrm{F}$. ou $145^{\circ} \mathrm{F}$. $\left(60^{\circ}\right.$ ou $62^{\circ} 7^{\mathrm{C}}$.) quand le couvercle du pasteurisateur était laissé ouvert et quand le robinet de sortie n'était pas nettoyé.

Conclusions. - Les résultats des études effectuées au laboratoire et dans des conditions industrielles indiquent que les organismes du genre Brucella sont détruits par les deux méthodes de pasteurisation quand les pasteurisateurs fonctionnent convenablement.

(A suivre.)

\title{
REVUE
}

\section{LA FABRICATION DU SUCRE DE LAIT}

\section{par G. GENIN}

Les compagnies laitières, ainsi que les fabriques de caséine, ont à leur disposition de grandes quantités de lait écrémé, qui sont susceptibles de constituer une source intéressante de lactose et dont l'utilisation correspond à un revenu additionnel pour cette industrie.

Le lait de vache renferme en solution vraie environ $4,7 \%$ de lactose. Lorsque, par fermentation, sa richesse en aeide lactique s'élève à $0,6 \%$, il coagule spontanément, mais le lactose que l'on obtient dans ces conditions, par eoncentration du sérum, n'est pas cristallin. On n'obtient des eristaux parfaitement formés, correspondant à Ia formule $\mathrm{C}^{12} \mathrm{H}^{22} \mathrm{O}^{11}+\mathrm{H}^{2} \mathrm{O}$, qu'en effectuant la eristallisation du lactose en partant de ses solutions aqueuses pures. Si les cristaux de lactose sont chauffés à $110-130^{\circ}$, ils perdent leur eau de cristallisation, et si on élève encore la température, le produit devient jaune, puis brun, et donne naissance à du lacto-caramel.

La préparation du lactose constitue donc une industrie tout à fait particulière, dont les caractéristiques ont été étudiées récemment dans la revue anglaise The Chemical Trade Journal, et nous avons emprunté à cette étude les renseignements qui suivent.

\section{L'influence de la fermentation lactique.}

Le lactose est insoluble dans l'alcool et dans l'éther, mais se 
dissout dans l'acide acétique chaud. Ses solutions sont facilement décomposées par les bactéries, et il peut se produire différents types de fermentation, la fermentation lactique étant la plus importante. Il est par conséquent indispensable, dans la fabrication du lactose, d'éviter la fermentation lactique dans le traitement du sérum. Dans la proportion d'environ $1 / 10^{\mathrm{e}}$ de molécule-gramme par litre, les sels des métaux alcalins et alcalino-terreux, retardent la fermentation lactique, mais les sels de fer, de magnésium et d'aluminium, sont à ce point de vue plus actifs, puisque un millième de moléculegramme par litre de ces sels suffit pour empêcher la fermentation.

Le sérum que l'on obtient après précipitation de la caséine par la présure diffère de celui obtenu par coagulation par les acides, par sa teneur en lactose et en acide lactique. Le premier renferme en effet de 4,5 à $5 \%$ de lactose, et des traces d'acide acétique, tandis que le seeond contient de 3,8 à $4 \%$ de lactose et jusqu'à $0,8 \%$ d'acide lactique. Les différents constituants du sérum ne présentent pas tous le même intérêt, et il est donc nécessaire de les doser séparément, en tenant compte des facteurs qui peuvent soit faciliter l'extraction du lactose, soit réduire sa teneur dans le sérum.

Un procédé qui donne d'excellents résultats pour éviter l'aotion des bactéries acidifiantes, consiste à refroidir le sérum à $12^{\circ}$. A cette température, en effet, l'action des bactéries est considérablement diminuée, mais elle réapparaît si on élève la température à $15^{\circ}$, pour atteindre un maximum lorsque la température est comprise entre 30 et $40^{\circ}$, diminuer ensuite et cesser presque complètement lorsque la température est comprise entre 45 et $50^{\circ}$. Une autre méthode consiste à ajouter à la solution des substances toxiques pour les bactéries, à condition que ces substances puissent être éliminées par la vapeur au cours de la concentration du sérum, quí se fait dans le vide. On utilise par exemple dans ce but une solution de formaline à $40 \%$ dans la proportion de 0,01 à $0,025 \%$ ou encore une solution de bisulfites de magnésium ou de sodium. Un point essentiel pour obtenir de bons résultats dans la fabrication du lactose est d'employer du sérum parfaitement frais. Si le sérum est sûr, sa teneur en lactose est réduite dans des proportions telles, que l'extraction de ce produit ne présente plus aucun intérêt économique.

L'élimination des matières grasses, des albuminoïdes et des sels qui subsistent dans le sérum, présente beaucoup plus de difficultés que celle de l'eau. Les matières grasses sont éliminées en premier lieu; si leur proportion est suffisante, on peut opérer par centrifugation, afin de les recueillir. Si la proportion de ces graisses est insuffisante, on peut l'élever en procédant à une évaporation partielle jusqu'à ce que la teneur en crème soit telle, qu'une centrifugation présente de l'intérêt. L'élimination des albuminoïdes, des sels et 
des traces de graisse qui subsistent après centrifugation, s'effectue sur le sérum très concentré ou sur le sirop épais que l'on obtient lorsque la majeure partie de l'eau a été évaporée.

\section{Séparation des protéines du sérum.}

La séparation et l'élimination complète des protéines présente de nombreuses difficultés. Les méthodes généralement employées conduisent en effet à la production d'un coagulum finement divisé, de telle sorte que la filtration est difficile et lente et que les pores du filtre sont rapidement colmatés par le dépôt de protéines. Il faut donc coaguler ces matières albuminoïdes afin de les rassembler en une forme facilement filtrable. Lorsque ces albuminoïdes sont éliminées, la principale cause de la fermentation lactique est supprimée, et on évite toutes les filtrations ultérieures laborieuses.

Pratiquement, on commence par neutraliser le sérum, pour le filtrer ensuite, afin de séparer complètement les protéines coagulées en suspension. L'excès d'aciđité est alors neutralisé avec du carbonate de calcium ou de baryum, ce dernier étant préférable, car il forme un sulfate moins soluble. Les liquides ainsi traités, sont ensuite stérilisés dans des autoclaves spéciaux de fer galvanisé, qui ont été préalablement stérilisés au moyen de formaline gazeuse. Le chauffage s'effectue par passage de vapeur soit dans un serpentin, soit dans un double fond. L'autoclave est muni d'un agitateur qui est mis en marche pendant toute la durée de l'opération et qui est généralement protégé par un rêvêtement isolant. La température maximum à laquelle on porte le liquide ne doit pas dépasser $125^{\circ}$, car il pourrait se produire une coloration de la solution.

Au bout de 20 minutes de chauffage, on laisse diminuer la pression jusqu'à ce que le liquide commence à bouillir, et on laisse cette ébullition se poursuivre jusqu'à ce que les albumines soient complètement coagulées et précipitées sous une forme granulaire que l'on peut facilement séparer par filtration, siphonage ou décantation. Le liquide est ensuite filtré dans un filtre à poche, afin d'éliminer totalement les matières en suspension, et les albumines coagulées recueillies au cours de cette opération, sont, après avoir été comprimées en gâteau, utilisées comme engrais.

Le filtrat que l'on obtient dans ces conditions ne contient done plus que du lactose, les sels du lait et ceux qui lui ont été ajoutés au cours du traitement précédent. La concentration de ce filtrat s'effectue dans le vide, et l'opération est arrêtée lorsque la liqueur a atteint une densité de 30 à $32^{\circ}$ Bé, correspondant à une concentration de $60 \%$. Il est nécessaire de vider le contenu des appareils à concentration avant que les solutions ne soient refroidies, ce qui entraînerait l'augmentation considérable de leur viscosité. Ces solutions 
sont versées dans des cristallisoirs de construction spéciale, et abandonnées au refroidissement. Dans certaines usines, ces cristallisoirs sont constitués par des cuves en fer rectangulaires placées dans d'autres cuves plus grandes parcourues par un courant d'eau froide. La masse est agitée, en même temps qu'elle épaissit lentement, et au bout de 24 heures, on peut séparer une masse granulaire et une couche superficielle huileuse.

Les cristaux de lactose sont séparés de la masse cuite par centrifugation. Les appareils que l'on utilise à cet effet diffèrent des appareils centrifuges employés pour l'écrémage par le fait qu'ils travaillent d'une façon discontinue, et à une plus faible vitesse. Lorsque le bol du centrifuge est complètement rempli de cristaux, on lave cette masse par un jet d'eau froide et on diminue graduellement la vitesse de l'appareil. On obtient ainsi du lactose brut, de coloration jaune clair, contenant encore de 10 à $15 \%$ d'impuretés (albumine, sels, etc.) et qui correspond aux $2 / 3$ du lactose contenu dans le sérum. Le sirop riche qui s'est séparé par centrifugation a une densité d'environ $15^{\circ}$ Bé, il renferme le $1 / 3$ restant du lactose, ainsi qu'une proportion importante d'impuretés qui empêchent la cristallisation de ce lactose.

\section{Purification des cristaux de lactose.}

Le lactose préparé comme il vient d'être dit, ne se présente pas sous une forme marchande. Il doit être raffiné, et l'opération s'effectue soit dans des raffineries -spéciales, soit dans les ateliers mêmes de séparation du lactose brut; dans ce cas, le produit n'a pas besoin d'être séché avant d'être raffiné.

Pour décolorer le lactose, et faire disparaître les goûts et les odeurs désagréables du produit, on ajoute à sa solution des charbons activés ou d'autres terres décolorantes. En même temps, les matières albuminoïdes qui sont encore présentes sont précipitées par l'addition de 200 grammes d'acide acétique pour 100 kilogrammes de solution de lactose à la température de $90^{\circ}$ C. Pour éliminer l'acide phosphorique, on ajoute alla solution un peude sulfate de magnésium, et elle est alors chauffée, jusqu'à ce que les produits précipités se séparent du liquide sous forme de grandes paillettes de produit. On filtre le sirop, alors qu'il est encore chaud, en utilisant des filtrespresses de grande surface.

Les impuretés recueillies sur le filtre sont lavées à l'eau sous pression, afin d'éliminer tout le lactose qu'elles contiennent, et les gâteaux comprimés sont ensuite traités avec de l'acide sulfurique, afin d'être transformés en un produit qui constitue un excellent engrais, par suite de sa haute teneur en azote et en phosphate assimilable. La solution de sucre limpide qui s'écoule du filtre est à 
nouveau concentrée dans le vide à une densité de $35^{\circ}$ Bé et dirigée, alors qu'elle est encore bouillante, dans le eristallisoir. La cristallisation demande facilement plusieurs jours, et on sépare d'abord les cristaux les plus fins par centrifugation. Un deuxième, puis un troisième passage au centrifuge, donnent des produits de moindre pureté.

Ces produits doivent subir un nouveau raffinage, afin d'être transformés en poudre blanche, qui constitue le lactose raffiné du commerce. A cet effet, le sucre de lait est dissous dans la quantité d'eau suffisante pour obtenir un sirop d'une densité de $15^{\circ}$ Bé. On fait bouillir ce sirop avec de petites quantités de sulfate d'aluminium ou de chlorure de calcium, afin de précipiter les matières colloïdales telles que albumines, par exemple. On passe encore au filtre-presse et le liquide limpide est concentré dans le vide, jusqu'à ce que sa densité s'élève à $22^{\circ}$ Bé. Par centrifugation de ce sirop, on obtient un produit blane qui est séché soit dans des cylindres rotatifs étamés, soit dans des dessiceateurs à air chaud. Après refroidissement, ce produit est broyé dans des broyeurs en porcelaine, puis il est tamisé dans des tamis à farine et exposé à l'air pendant quelques jours.

Les prineipaux défauts des échantillons de lactose sont la présence d'une proportion excessive de gros grains, une humidité trop élevée, une coloration jaunâtre, l'existence d'impuretés organiques et la présence de sels de métaux lourds. Une trop grande humidité et la présence de gros grains découlent d'un soin insuffisant apporté à la fabrication. La coloration jaune peut être attribuée, soit à un lavage incomplet, soit à un séchage à une température trop élevée. Enfin, la présence de matières organiques ou inorganiques résulte de l'emploi de filtres insuffisants.

\section{Les emplois du sucre de lait.}

Dans l'industrie pharmaceutique, le lactose raffiné est employé dans un grand nombre de préparations, dont les principales sont celles des aliments diététiques contenant $25 \%$ de cacao pur, des mélanges de sucre de lait et de cacao contenant une proportion élevée de fer assimilable, du bouillon Liebig et des aliments similaires pour enfants, riches en lactose et en vitamines. On trouve décrit dans un grand nombre de brevets ayant trait à la fabrication d'aliments spéciaux, l'emploi du lactose. L'addition de petites quantités de lactose aux pâtes pour pain et pour biscuits, augmente la valeur alimentaire de ces produits. Il est également employé dans la fabrication du chocolat, auquel il communique un goût spécial, dans la fabrication des amandes sucrées, dans la préparation des conserves de fruits, d'olives, et de citrons, etc. Dans l'industrie des 
confitures, le lactose remplace avec avantage certains sucres dont la fermentation est beaucoup plus facile. Il joue un rôle important dans la conservation des fruits légèrement acidulés et on a constaté $q u$ 'il assure une meilleure conservation de l'état des fruits à chair molle, telles les fraises, cerises, etc. En effet, par suite de la transformation qu'il subit, il donne naissance à une faible proportion d'acide lactique, quiaugmente la résistance de ces fruits à l'action des agents de conservation, et réduit leur décoloration.

L'emploi de faibles proportions de lactose, de 1 à $2 \%$, permet de remplacer le sel, le salpêtre, etc., dans la préparation des conserves de porcs et assure une bonne conservation de la saveur, de la coloration et de la consistance de ces produits. Le lactose et les petites quaritités d'acide lactique auxquelles il donne naissance peuvent être employés pour la protection des conserves de viande et sont supérieurs aux nitrites au point de vue hygiénique.

Le lactose est également utilisé pour assurer la conservation des tourteaux que l'on obtient dans la préparation de l'huile de coprah, de tournesol, etc., et qui contiennent des huiles et des matières grasses dont la fermentation est facile. Le lactose subit en effet dans ces produits une légère fermentation et l'acide lactique qui prend naissance protège les huiles et matières grasses contre l'oxydation en s'opposant aux lipases oxydants, telle l'oléase. Ces tourteaux deviennent donc plus appétissants pour les animaux et d'une digestion plus facile. Le lactose accélère le démarrage et facilite la fermentation lactique de certains produits conservés en silos. Il favorise la concentration des jus de fruits tels que oranges, citrons, etc., dans le vide et est un excellent agent de conservation des essences de fruits faciles à oxyder. Le lactose a été employé dans certaines proportions comme agent de conservation du latex de caoutchouc, par suite de son action réductrice et du fait qu'il s'oppose à la résinification. Dans l'industrie du savon, il stabilise les matières colorantes naturelles organiques, comme la chlorophylle, et la transparence des savons. Il peut être employé comme agent réducteur dans la fabrication des miroirs et comme stabilisant dans la préparation de certains explosifs. 